ARTICLE

\title{
Structures of the human mitochondrial ribosome bound to EF-G1 reveal distinct features of mitochondrial translation elongation
}

\author{
Ravi Kiran Koripella (1) 1,7, Manjuli R. Sharma (10 1,7, Kalpana Bhargava2,4, Partha P. Datta1,5, Prem S. Kaushal ${ }^{1,6}$, \\ Pooja Keshavan', Linda L. Spremulli², Nilesh K. Banavali id ${ }^{1,3}$ \& Rajendra K. Agrawal (1) 1,3凶
}

The mammalian mitochondrial ribosome (mitoribosome) and its associated translational factors have evolved to accommodate greater participation of proteins in mitochondrial translation. Here we present the 2.68-3.96 $\AA$ cryo-EM structures of the human $55 \mathrm{~S}$ mitoribosome in complex with the human mitochondrial elongation factor $\mathrm{G} 1$ (EF-G1 $1_{\mathrm{mt}}$ ) in three distinct conformational states, including an intermediate state and a post-translocational state. These structures reveal the role of several mitochondria-specific (mito-specific) mitoribosomal proteins (MRPs) and a mito-specific segment of $\mathrm{EF}-\mathrm{G} 1_{\mathrm{mt}}$ in mitochondrial tRNA (tRNA $\mathrm{mt}_{\mathrm{m}}$ ) translocation. In particular, the mito-specific C-terminal extension in $\mathrm{EF}-\mathrm{G} 1_{\mathrm{mt}}$ is directly involved in translocation of the acceptor arm of the A-site tRNA $A_{m t}$. In addition to the ratchet-like and independent head-swiveling motions exhibited by the small mitoribosomal subunit, we discover significant conformational changes in MRP $\mathrm{mL} 45$ at the nascent polypeptide-exit site within the large mitoribosomal subunit that could be critical for tethering of the elongating mitoribosome onto the inner-mitochondrial membrane.

\footnotetext{
${ }^{1}$ Division of Translational Medicine, Wadsworth Center, New York State Department of Health, Empire State Plaza, Albany, NY 12201, USA. ${ }^{2}$ Department of Chemistry, Campus Box 3290, University of North Carolina, Chapel Hill, NC, USA. ${ }^{3}$ Department of Biomedical Sciences, University at Albany, SUNY, Albany, NY 12201-0509, USA. ${ }^{4}$ Present address: High Energy Material Research Lab, Defense Research and Development Organization, Sutarwadi, Pashan, Pune, Maharashtra 411021, India. ${ }^{5}$ Present address: Department of Biological Sciences, Indian Institute of Science Education and Research Kolkata, Mohanpur, West Bengal 741246, India. 'Present address: Regional Centre for Biotechnology, 3rd Milestone, Faridabad-Gurgaon Expressway, PO Box \# 3, Faridabad, Haryana 121001, India. ${ }^{7}$ These authors contributed equally: Ravi Kiran Koripella and Manjuli R. Sharma. ${ }^{\varpi}$ email: rajendra.agrawal@health.ny.gov
} 
$\mathrm{M}$ itochondria are thought to have originated through an early endosymbiotic event between an $\alpha$ protobacterium and a primitive eukaryotic host cell ${ }^{1}$. However, the structural, functional, and compositional organization of the mitochondrial ribosomes (mitoribosome) is dramatically different from its cytoplasmic and bacterial counterparts $^{2-7}$. The ribosomal RNA (rRNA) to ribosomal protein ratio in mammalian mitoribosome $(\sim 1: 2)$ is reversed as compared to that in the eubacterial ribosomes $(\sim 2: 1)$. The first cryo-EM study of the mammalian mitoribosome identified several unique structural features ${ }^{5}$ in both its subunits: the large 395 subunit (LSU) and the smaller $28 \mathrm{~S}$ subunit (SSU). Subsequent high-resolution structures ${ }^{2,4,8,9}$ provided molecular description of previously identified features ${ }^{5}$, such as heavily shielded rRNA cores by mitoribosomal proteins (MRPs), the presence of a significantly modified entrance of the mRNA channel and nascent polypeptide-exit tunnel (NPET), and a P-site finger. In addition, the high-resolution structures revealed that one of the mitochondrial tRNAs ( $\left(\mathrm{RNAs}_{\mathrm{mt}}\right.$ ) partially substitutes for the role of bacterial 5S rRNA by becoming a structural component of the mammalian mitoribosomal LSU2,4.

Similar to bacterial translation, the mechanism of the mammalian mitochondrial translation is roughly divided into four stages: initiation, elongation, termination, and ribosome recycling ${ }^{10,11}$. Each of these steps are facilitated by translational factors that are homologous to their bacterial counterparts but carry mitochondria-specific (mito-specific) segments ${ }^{10,11}$. Biochemical $^{12-15}$ and structural ${ }^{8,9,16,17}$ studies have shown that the mito-specific segments in translational factors play important functions in mitochondrial translation. The distinct structural features in both the mitoribosome and its binding translational factors therefore suggest unique molecular interactions and mechanism during each mitochondrial translation step. The critical step of tRNA and mRNA translocation on the ribosome is promoted by elongation factor-G (EF-G) in eubacteria and homologous EF-2 in eukaryotic cytoplasm. In mammalian mitochondria, there are two isoforms of $\mathrm{EF}-\mathrm{G}_{\mathrm{mt}}: \mathrm{EF}-\mathrm{G} 1_{\mathrm{mt}}$ and EF-G2 $2_{\mathrm{mt}}^{18,19}$. EF-G1 $1_{\mathrm{mt}}$ catalyzes tRNA $\mathrm{mt}_{\mathrm{mt}}$ translocation on the mitoribosome, whereas $\mathrm{EF}-\mathrm{G} 2_{\mathrm{mt}}$ is involved exclusively in mitoribosome recycling ${ }^{19}$. A mutation in human $\mathrm{EF}-\mathrm{G} 1_{\mathrm{mt}}$ leads to fatal hepatoencephalopathy, indicating that this isoform is essential for mitochondrial protein biosynthesis in humans ${ }^{20,21}$. In addition, defects in mitochondrial protein synthesis are associated with numerous human diseases that directly involve mutations in MRPs and tRNAs $\mathrm{mt}_{\mathrm{mt}}^{22-24}$

The bacterial EF-G is composed of five structural domains, namely G (or domain I) and domains II - $\mathrm{V}^{25,26}$. The structural and functional aspects of EF-G-catalyzed translocation on bacterial ribosomes have been extensively studied in various functional states, using both cryo-EM ${ }^{27-33}$ and X-ray crystallography ${ }^{34-38}$. Like other translocases, the mammalian EF$\mathrm{Gl}_{\mathrm{mt}}$ (molecular weight $\sim 80 \mathrm{kDa}$ ) is a single polypeptide that possesses mito-specific extensions at both its termini with an additional 47 amino acids (aa), including the signal sequences, as compared to its bacterial homologs ${ }^{39}$. Human EF-G1 $1_{\mathrm{mt}}$ is 751 aa long, where the first 36 residues at the $\mathrm{N}$-terminus constitute the mitochondrial targeting signal ${ }^{39}$, which is cleaved off in the functional form. The functional human EF-G1 $1_{\mathrm{mt}}$ shows $\sim 45 \%$ sequence identity with its bacterial counterpart, with a major difference being the presence of an 11 aa mito-specific extension at its $\mathrm{C}$ terminus. We have determined near-atomic-resolution cryo-EM structures of the human $55 \mathrm{~S}$ mitoribosome in complex with the human EF-G1 $1_{\mathrm{mt}}$ to investigate the roles of the mitospecific MRPs and C-terminal extension in EF-G1 $1_{\mathrm{mt}}$ in $\mathrm{tRNA}_{\mathrm{mt}}$ translocation in mammalian mitochondria. Our study reveals several distinct features, including mito-specific molecular interactions during EF-G1 $1_{\mathrm{mt}}$-mediated $\mathrm{tRNA} \mathrm{mt}_{\mathrm{m}}$ translocation on the human mitoribosome. In addition, we identify conformational changes associated with translation elongation at the exit of the NPET within the mitoribosome that could be necessary for facilitating the release of the nascent polypeptide chain through the NPET and anchoring of the mitoribosome on to the inner mitochondrial membrane.

\section{Results and discussion}

Overall structure of the 55S.EF-G1 $1_{\mathrm{mt}}$ complex. To obtain the 55S.EF-G1 ${ }_{\mathrm{mt}}$ complex a non-hydrolysable analog of GTP, GMPPCP, was used to lock EF-G1 $1_{\mathrm{mt}}$ on the mitoribosomes (see Methods). A cryo-EM structure for the 55S.EF-G1 $1_{\mathrm{mt}}$ GMPPCP complex with an overall resolution of $2.7 \AA$ (Supplementary Figs. 1 and 2) was obtained (see Methods). 3D classification of all selected $55 \mathrm{~S}$ mitoribosome images yielded two major classes that were refined to $2.96 \AA$ and $2.97 \AA$ resolution, respectively, and a minor class that was refined to $3.96 \AA$ \& resolution (Supplementary Figs. 1 and 2; Supplementary Table 1). All three 55S mitoribosome maps show well-defined densities for EF-G1 $1_{\mathrm{mt}}$, but reveal significant differences in the relative orientation of the $28 \mathrm{~S}$ subunit with respect to the $39 \mathrm{~S}$ subunit. The cryo-EM structure with the $28 \mathrm{~S}$ subunit in its fully rotated state ${ }^{8}$ is referred to as Class I, the structure with the $28 \mathrm{~S}$ subunit in its unrotated canonical state $^{2,4}$ is referred to as Class III (Fig. 1a, b), and the structure with the $28 \mathrm{~S}$ subunit an intermediate state between the Class I and Class III conformations is referred to as Class II. Each of these structures shows variable densities for tRNAs $s_{\mathrm{mt}}$ in the mitoribosomal peptidyl (P) and exit (E) sites (Fig. 1a, b; and Supplementary Notes).

The overall conformation of $\mathrm{EF}-\mathrm{G} 1_{\mathrm{mt}}$ on the $55 \mathrm{~S}$ mitoribosome in all three maps is analogous to the structure of EF-G in the cytoplasmic ribosomal complexes, where the factor has been trapped either with the help of the antibiotic fusidic acid (FA) 27,31,32,35 or by using a non-hydrolysable GTP analog $29,33,34,36,37$. In addition to determining the structure of complete EF- $G 1_{\mathrm{mt}}$ with all of its 715 aa residues that fold into five globular domains (Fig. 1c), high-resolution features in our cryo-EM maps (Supplementary Fig. 3) allow us to model 75 rRNA residues and 1,082 aa residues that are absent in the currently available human mitoribosome structure ${ }^{2}$. Furthermore, we identify species-specific structural differences among mammalian MRPs. The EF-G1 $1_{\mathrm{mt}}$ binding stabilizes the flexible C-terminal domain (CTD) of the uL10-L12 stalk ${ }^{29}$ and enables modeling of one copy of the uL12m CTD that interacts with the $\mathrm{G}^{\prime}$ subdomain of EF$\mathrm{G} 1_{\mathrm{mt}}$ (Fig. 1d). EF-G1 $1_{\mathrm{mt}}$ induced movement also brings the Nterminal domain (NTD) of uL $11 \mathrm{~m}$ by $5 \AA$ closer to the uL12mCTD (Supplementary Fig. 4c), thereby enabling the latter to simultaneously interact with the $\mathrm{uL} 11 \mathrm{~m}$ and the $\mathrm{G}^{\prime}$ subdomain to form an arc-like structure ${ }^{27,29}$. A direct interaction between $\mathrm{uL} 12 \mathrm{~m}$ CTD and EF-G1 $1_{\mathrm{mt}}$ suggests uL12m's role in factor recruitment ${ }^{40,41}$ during mitochondrial translation.

Interactions of EF-G1 $1_{\mathrm{mt}}$ with the GTPase-activating center of the mitoribosome. Translocation of tRNAs and mRNA is an intrinsic property of the ribosome but binding of EF-G.GTP and subsequent hydrolysis of GTP on EF-G enhances the rate of translocation by several orders of magnitude ${ }^{42,43}$. Using our higher resolution map (Complex III), a complete de novo model of the nucleotide-binding pocket and the interactions of switch I with other domains of EF-G1 $1_{\mathrm{mt}}$ and the adjacent ribosomal components could be constructed. The bound GMPPCP is held in position through a large network of hydrogen bonds and van der Waals interactions with several highly conserved EF-G1 $1_{\mathrm{mt}}$ residues, notably D56 and K59 from the P-loop, T101 from the 
a

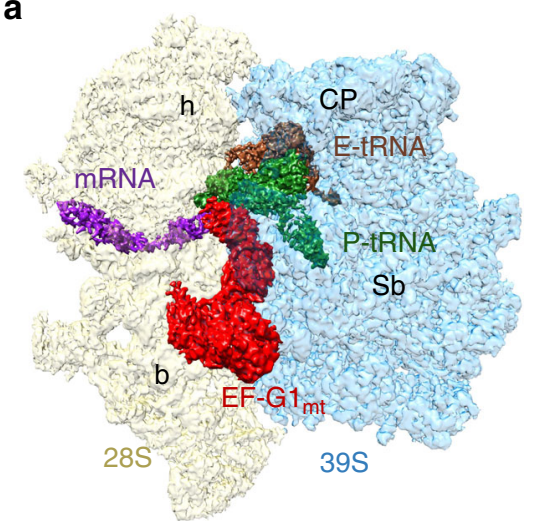

C

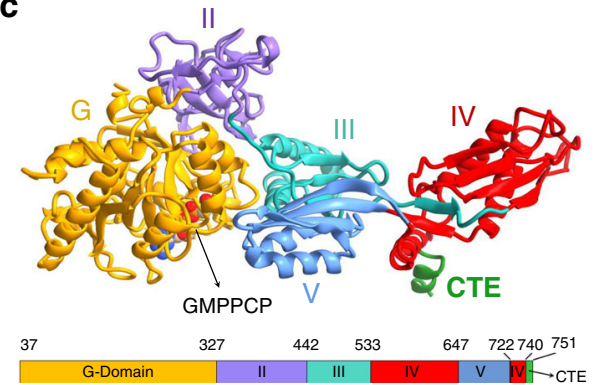

b

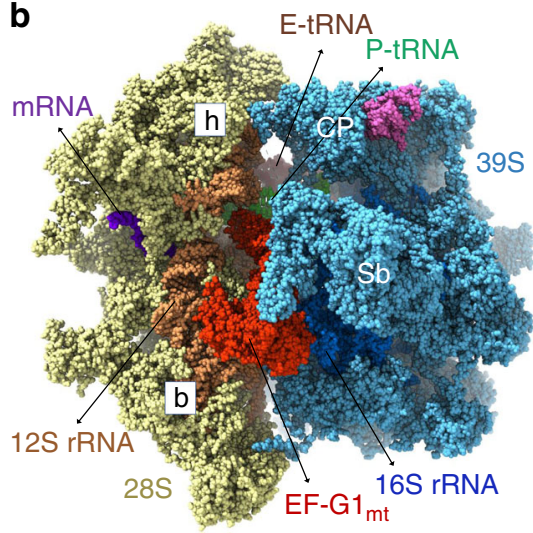

d

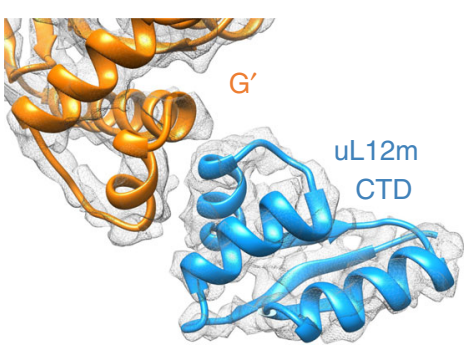

Fig. 1 Cryo-EM structure of the human mitochondrial 55S-EF-G1 $\mathbf{m i t}_{\mathbf{m}} \cdot \mathbf{G M P P C P}$ Class III complex. a Segmented cryo-EM densities, showing 28S

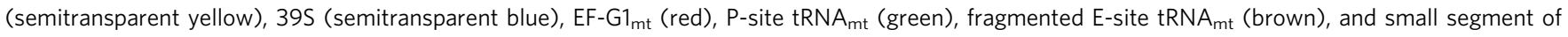
mRNA (purple), as viewed from the subunit-subunit interface side. $\mathbf{b}$ Atomic model of components within the complex, colored as in panel $\mathbf{a}$. Landmarks on the $28 \mathrm{~S}$ subunit: $\mathrm{h}$ head, $\mathrm{b}$ body. Landmarks on the $39 \mathrm{~S}$ subunit: CP central protuberance, Sb uL11m stalk base. c Structure of EF-G1 $\mathrm{mt}$ displaying its five domains and the bound GMPPCP molecule, with a color-coded bar diagram depicting domain organization of EF-G1 $\mathrm{mt}_{\text {. }} \mathbf{d}$ Interaction between the C-terminal domain (CTD, blue) of $\mathrm{uL} 12 \mathrm{~m}$ and $\mathrm{G}^{\prime}$ subdomain (orange) of $\mathrm{EF}-\mathrm{G} 1_{\mathrm{mt}}$.

switch I region, and $\mathrm{H} 124$ from the switch II region (Fig. 2a). A crucial $\mathrm{Mg}^{2+}$ ion positioned close to $\gamma$ phosphate of GMPPCP is coordinated by T60 from the P-loop and T101 from the switch I region (Fig. 2b). H124 is known to be essential for catalyzing the hydrolysis of GTP, as mutation of this crucial residue in bacterial EF-Tu severely inhibits its ribosome-stimulated GTP hydrolysis $^{44,45}$. In our maps, the catalytic $\mathrm{H} 124$ is oriented towards the $\gamma$ phosphate of the bound GMPPCP molecule (Fig. 2c), representing an active nucleotide-binding pocket ${ }^{34,36}$ while its analog is pointed in the opposite direction in a GDP-state bacterial post-translocation complex (Supplementary Fig. 5$)^{35}$. The positioning of H124 is stabilized by the universally conserved sarcin-ricin loop (SRL) of the 16S rRNA segment from the 39S LSU, which plays a central role in activating the translational GTPases ${ }^{34-36}$. Binding of $G$ domain adjacent to the SRL closes the nucleotide-binding pocket of EF-G1 $1_{\mathrm{mt}}$ and the base A3129 from the SRL is responsible for stabilizing the current activated conformation of $\mathrm{H} 124$ through hydrogen-bonding interactions (Fig. 2c, d). Bases A3130 and G3131 from the SRL are found coordinating a uniquely placed $\mathrm{Mg}^{2+}$ ion which in turn is known to stabilize the P-loop D56 in an active conformation (Fig. 2c, d). As observed in bacteria ${ }^{34,36}$, the binding of EF-G1 1 to the $55 \mathrm{~S}$ mitoribosome stabilizes the factor in an active conformation necessary to catalyze the hydrolysis reaction, by ordering the switch I region and positioning of D56 and H124 towards the active site for GTP hydrolysis.

Interactions of domain IV of EF-G $1_{m t}$ in the A site of the $28 \mathrm{~S}$ subunit in three conformational states. In all three $55 \mathrm{~S} \cdot \mathrm{EF}-\mathrm{G} 1_{\mathrm{mt}}$ complexes, the EF-G1 $1_{\mathrm{mt}}$ is held in position by interacting with several components of both the large and small mitoribosomal subunits but domain IV of EF-G1 $1_{\mathrm{mt}}$ and mitoribosomal components interact differently among these complexes. In the Class I $55 \mathrm{~S} \cdot \mathrm{EF}-\mathrm{G} 1_{\mathrm{mt}}$ complex, the $28 \mathrm{~S}$ subunit has undergone a $\sim 9.5^{\circ}$ counter-clockwise rotation relative to the $39 \mathrm{~S}$ subunit (Fig. 3a), similar to previously described ratchet-like inter-subunit reorganization of the bacterial ribosome $e^{30,46}$. In addition to this ratchetlike motion, significant head swiveling ${ }^{32,47}$ was also observed in the Class I complex, where the $28 \mathrm{~S}$ subunit head region has rotated $\sim 3^{\circ}$ relative to the body in a roughly orthogonal direction to the ratchet-like motion (Fig. 3a). The Class II complex presents a previously unknown EF-G-bound conformational intermediate, where the head region of the $28 \mathrm{~S}$ subunit has swiveled as in the ratcheted Class I complex (Fig. 3b), while the conformation of the $28 \mathrm{~S}$ body region is similar to that in the unrotated Class III complex (Fig. 3c). The presence of a Class I complex-like conformational state with ratcheted and head rotated SSU in a previous $E F-G 1_{m t}$-unbound map $^{2}$ but the absence of such a conformation in our control maps (Supplementary Fig. 6) suggest that the Class I complex is formed either upon binding of EF$\mathrm{G} 1_{\mathrm{mt}}$ to a subpopulation of mitoribosomes that carries only a single tRNA at the E-site 2,48 (Fig. 3d), or to a population that carries loosely bound P-site tRNAs that are all translocated to the E-site. Overall, we find that EF-G1 $1_{\mathrm{mt}}$ binding brings a greater proportion of mitoribosomes into unratcheted state, when compared with the distribution in our control population (Supplementary Fig. 6). The Class II complex represents the smallest of the three populations and shows a strong density for the E-site tRNA but a somewhat fragmented tRNA density at the $\mathrm{P}$ site (Fig. 3e), whereas the Class III complex shows densities for both P- and E-site tRNAs (Figs. 1a, 3f). 
a

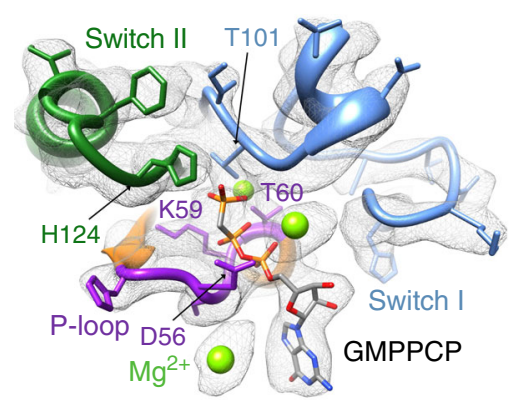

C

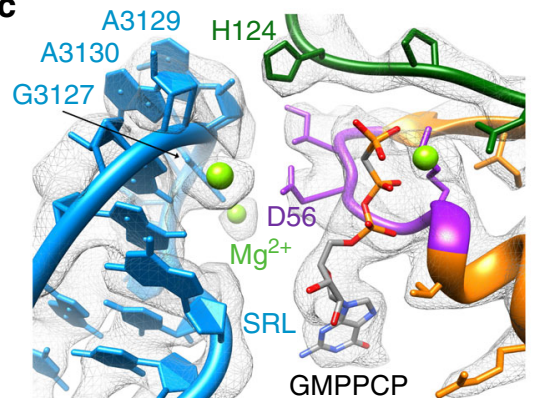

b

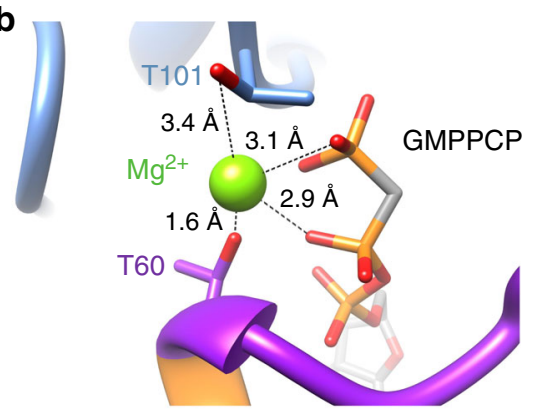

d

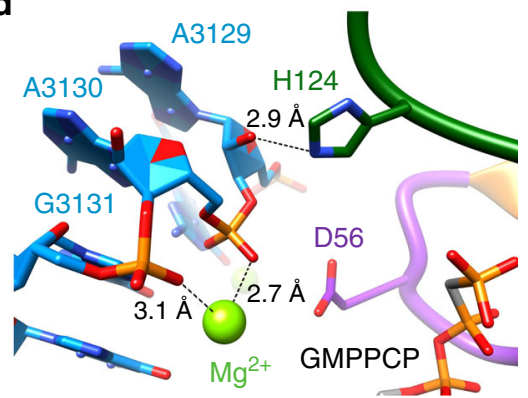

Fig. 2 Interactions of GMPPCP and $\mathbf{M g}^{\mathbf{2}+}$ within the $\mathbf{G}$ domain of $\mathbf{E F}-\mathbf{G 1}_{\mathbf{m t}}$. a GMPPCP position in the nucleotide-binding pocket through multiple interactions with conserved aa residues of functionally essential elements of the G domain such as Switch I (blue), Switch II (green), and the P-loop (purple). The bound magnesium ions are shown as green spheres. b T60 from the P-loop and T101 from the switch I region coordinates a crucial $\mathrm{Mg}^{2+}$ ion near GMPPCP. c Interactions of H124 (green) and D56 (purple) with the functionally important bases from the $\alpha$-sarcin-ricin loop (SRL): A3129, A3130, and G3131. d H124 is stabilized in its active conformation through interactions with the sugar moiety of a highly conserved residue of the SRL A3129. Phosphate backbones from SRL's A3130 and A G3131 residues coordinate a Mg ${ }^{2+}$ ion that in turn stabilize D56 in its active conformation. Colors of G domain components in panels a-d are matched.

Domain IV of the bacterial EF-G is known to play a crucial role in tRNA and mRNA translocation ${ }^{27,49}$. In all three Classes, Domain IV of the EF-G1 $1_{\mathrm{mt}}$ is inserted into the $28 \mathrm{~S}$ subunit decoding center such that it would sterically overlap with the anticodon arm of an A-site tRNA (Fig. 3g-i), as found in case of analogous bacterial complex ${ }^{27}$. Minimum mitoribosomal interactions with domain IV occur in Class I complex, an intermediate number of interactions occur in Class II complex, and the maximum interactions occur in Class III complex (Fig. 3g-i). In the Class III complex, Domain IV makes contacts with multiple $12 \mathrm{~S}$ rRNA components of the $28 \mathrm{~S}$ subunit such as helix 24 (h24), $\mathrm{h} 30, \mathrm{~h} 44$, and the anticodon arm of the tRNA bound in the P/P state (Fig. 3i). [We have adopted the bacterial numbering to refer to rRNA helices throughout, as they are identified by a number prefixed with an ' $h$ ' for the mitochondrial $12 \mathrm{~S}$ rRNA in SSU and an ' $\mathrm{H}$ ' for its $16 \mathrm{~S}$ rRNA in LSU. The rRNA nucleotide numbering are according to Amunts and coworkers ${ }^{2}$.] In the 28S P-site region, aa residues S543, G544 and G545 from the loop1 region of domain IV interact with the backbone phosphates of h44 bases C1561 and G1562, while base A1078 from h24 is placed within hydrogen-bond forming distance from G544 and G545 of domain IV (Fig. 3i). The Class II and Class III maps show density for a Psite tRNA (Fig. 3e, f) bound in the classical P/P state ${ }^{4,5}$. However, the P-site density is relatively weak, because it represents an averaged density of the endogenously bound multi-sized tRNAs $_{\mathrm{m}}$, some of which are known to have much smaller T-loops compared to their bacterial counterparts ${ }^{50,51}$. Nevertheless, conserved segments such as anticodon and CCA arm of tRNA could be docked into corresponding densities. Accordingly, nucleotides 33 and 34 from the anticodon of the P-site tRNA are positioned within $3 \AA$ of residues M618 and V619 (Fig. 3i) from the loop 3 region of domain IV. In the $28 \mathrm{~S}$ head region of Class III complex, the backbone phosphates of the 12S rRNA bases U1209 and U1210 interact with aa residues S576 and N577 from the loop2 region of domain IV (Fig. 3i). The size variability in $\mathrm{tRNAs}_{\mathrm{mt}}$ also affect the density corresponding to the anticodon region of the E-site $\mathrm{tRNA}_{\mathrm{mt}}$ (Supplementary Notes and Supplementary Fig. 7).

Simultaneous interactions of domain IV with both the head and shoulder regions of the $28 \mathrm{~S}$ subunit and the anticodon region of the tRNA in Complex III would stabilize the tRNA in the P site and prevent the anticodon end of translocated P-site tRNA from slipping back to the A site, as also suggested by structural studies on bacterial translocation $31,32,35,52$. The small subunit of a bacterial $70 \mathrm{~S}$ ribosome is also found in an unrotated conformation with similar interactions in the crystallographic structure of the 70S.EF-G.GDP.FA post-translocational complex ${ }^{35}$, suggesting that our Class III 55S.EF-G1 $1_{\mathrm{mt}}$ complex represents an authentic post-translocation state of the human mitoribosome. The core $12 \mathrm{~S}$ SSU rRNA regions of the mitochondrial and bacterial ribosomes that are known to interact with A- and P-site tRNAs in eubacteria are generally conserved ${ }^{5,53}$, and the relative orientations of bound A- and P-site tRNAs are also similar (Supplementary Fig. 8), despite the presence of a significantly altered and MRP-enriched environment around the tRNA binding sites in the LSU of the mitoribosome as elaborated under the next heading.

The tip of EF-G1 $\mathrm{mt}$ domain IV in the Class I complex is positioned $\sim 10 \AA$ away from 12S rRNA helices h24 and h30, closer to the $28 \mathrm{~S}$ shoulder or the A site than its position in the Class III complex (Fig. 3g, also see Supplementary Fig. 9). Domain IV does not interact with the $28 \mathrm{~S}$ head region in Class I complex, and the only 28S subunit element that still interacts with domain IV is the $12 \mathrm{~S}$ rRNA h44 (Fig. $3 \mathrm{~g}$ ). This is not surprising since simultaneous interactions of domain IV with both the head and platform components would impede the head rotation, and a 
a

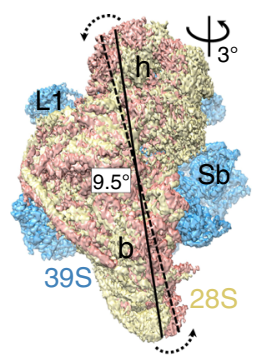

d

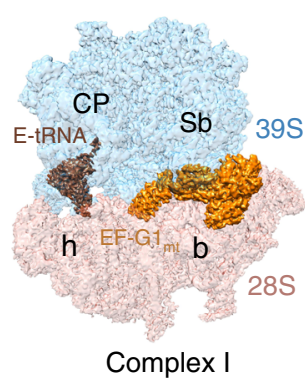

g

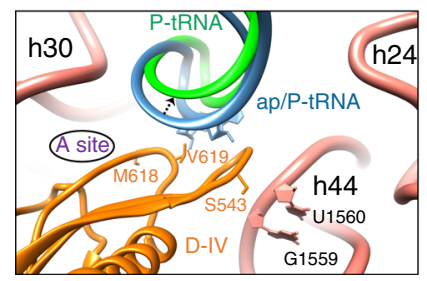

b

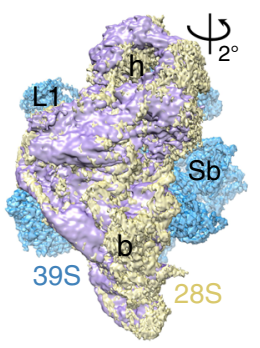

e

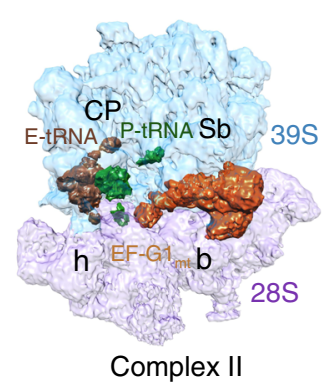

h

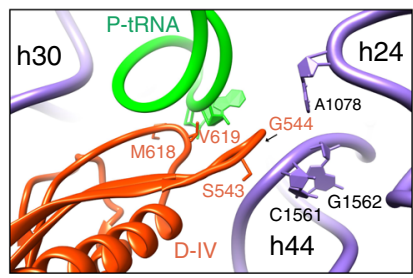

C

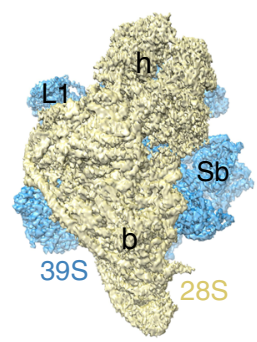

f

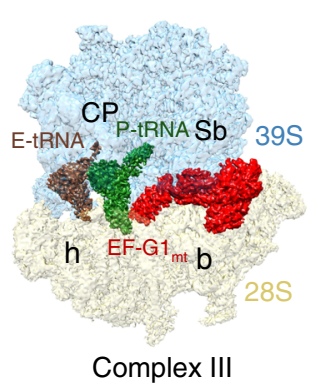

i

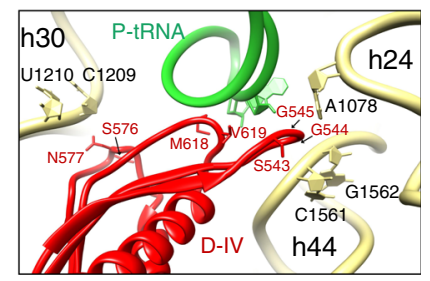

Fig. 3 Conformational states of the $\mathbf{2 8 S}$ subunit and interactions of domain IV of EF-G1 $\mathbf{m}_{\mathbf{m t}}$ in $\mathbf{5 5 S} \cdot \mathbf{E F}-\mathbf{G 1}_{\mathbf{m t}} \cdot \mathbf{G M P P C P}$ complexes. a-c Intersubunit rotation and the $28 \mathrm{~S}$ head swiveling. a Superimposition of the cryo-EM maps of the Class I and the Class III complexes revealed an overall $9.5^{\circ}$ rotation of the $28 \mathrm{~S}$ subunit (salmon) in an anti-clockwise direction relative to the $39 \mathrm{~S}$ subunit (blue) in Class I complex, with an additional rotation of the $28 \mathrm{~S}$ head domain by $\sim 3^{\circ}$ in a roughly orthogonal direction to the direction of the inter-subunit rotation. b Similar comparison between the Class II and Class III complexes revealed $\sim 2^{\circ}$ rotation of the $28 \mathrm{~S}$ head in the Class II complex (light purple), without a measurable overall inter-subunit rotation. In all three panels, the $55 \mathrm{~S}$ mitoribosome is shown from the $28 \mathrm{~S}$ solvent side. The $28 \mathrm{~S}$ subunit from Complexes I and II are shown in salmon and purple colors, whereas that from the Class III complex is shown in yellow color. Landmarks for both mitoribosomal subunits in panels a-f are the same as introduced in Fig. 1, except for L1 stalk

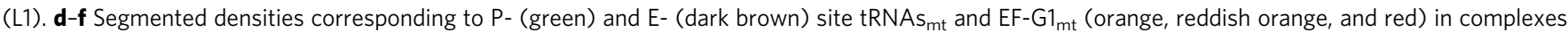
I-III are shown, with semitransparent densities for the $28 \mathrm{~S}$ (bottom) and $39 \mathrm{~S}$ (top) subunits. g-i Interactions of EF-G1 $\mathrm{mt}$ domain IV with the $28 \mathrm{~S}$ subunit and the P-site tRNA. $\mathbf{g}$ In the Class I complex, domain IV (orange) is positioned 10 $\AA$ away from the $12 \mathrm{~S} \mathrm{SSU}$ rRNA helices (salmon) h30 and h24. A 6-7 $\AA$ shift in the anticodon end of P-tRNA (green) to an ap/P hybrid position (blue) is required to establish contacts with the EF-G1 $\mathrm{mt}$ domain IV (orange). The A-site location is indicated. $\mathbf{h}$ Intermediate head swiveled-only state shows limited interactions of domain IV (reddish orange) with the 12S rRNA helices (light purple) h24 and h44. i Multiple interactions of domain IV (red) with the 12S rRNA (yellow) and P-site tRNA (green) in the Class III complex.

combination of subunit ratcheting and head swiveling help translocate the $\mathrm{A}$ - and $\mathrm{P}$-site tRNAs into the $\mathrm{P}$ and $\mathrm{E}$ sites, respectively ${ }^{31,32,47}$. Unlike the Class III complex, the Class I map does not have enough density to model a P-site tRNA but superimposing the Class III P-site tRNA density onto the Class I complex suggests that the interactions of domain IV with anticodon of the P-site tRNA in the $\mathrm{P} / \mathrm{P}$ state would not be established in the Class I complex (Fig. 3g). Interestingly, these contacts can be restored if the anticodon end of the P-site tRNA is moved by 6-7 $\AA$ towards the A site of the $28 \mathrm{~S}$ subunit (Fig. $3 \mathrm{~g}$ ), which indicates that the Class I 55S.EF-G1 $1_{\mathrm{mt}}$ complex represents a key early translocation intermediate where the domain IV has moved only partially into the A site following the movement of A-site tRNA into an intermediate chimeric ap/P state ${ }^{31}$, preceding the Class II state (Fig. $3 \mathrm{~h}$ ) that is followed by the Class III state (Fig. 3i). Domain IV of EF-G1 $1_{\mathrm{mt}}$ synchronizes the ratcheting motion of the ribosome along with the movement of tRNAs, as it appears to closely follow the anticodon arm of the Asite tRNA during its translocation into the $\mathrm{P}$ site $31,32,35,52$.
Role of P-site finger and other MRPs that directly interact with $\mathbf{t R N A s}_{\mathbf{m t}}$ and EF-G1 $\mathbf{1}_{\mathbf{m t}}$. One of the major structural differences between the bacterial and mitochondrial ribosomes is the loss of several rRNA segments in mitochondria that are partially compensated by the acquisition of new MRPs and extensions in homologous MRPs ${ }^{5,54-56}$. This also changes the compositional landscape of the ribosomal intersubunit space that provides the corridor for the mRNA and tRNA movement on the mammalian mitoribosome during translation elongation. In mammalian mitochondria, protein bL5 is lost from the P site while bL25 and the A-site finger (23S rRNA helix 38) are lost from the A site ${ }^{2,4,5}$. The loss of these structural elements that interact with bound tRNA molecules is compensated by a unique finger-like structural element called the P-site finger (PSF) that interacts with both the A- and P-site bound tRNAs ${ }^{4,5}$. In the Class III complex, the PSF is found interacting with both the T-loop and the D-loop of P-site tRNA (Fig. 4a). The role of PSF appears to be to correctly position the A- and P-site tRNAs and prevent the elbow region of the $\mathrm{P}$-site bound tRNA from reverting back to the A site during and 

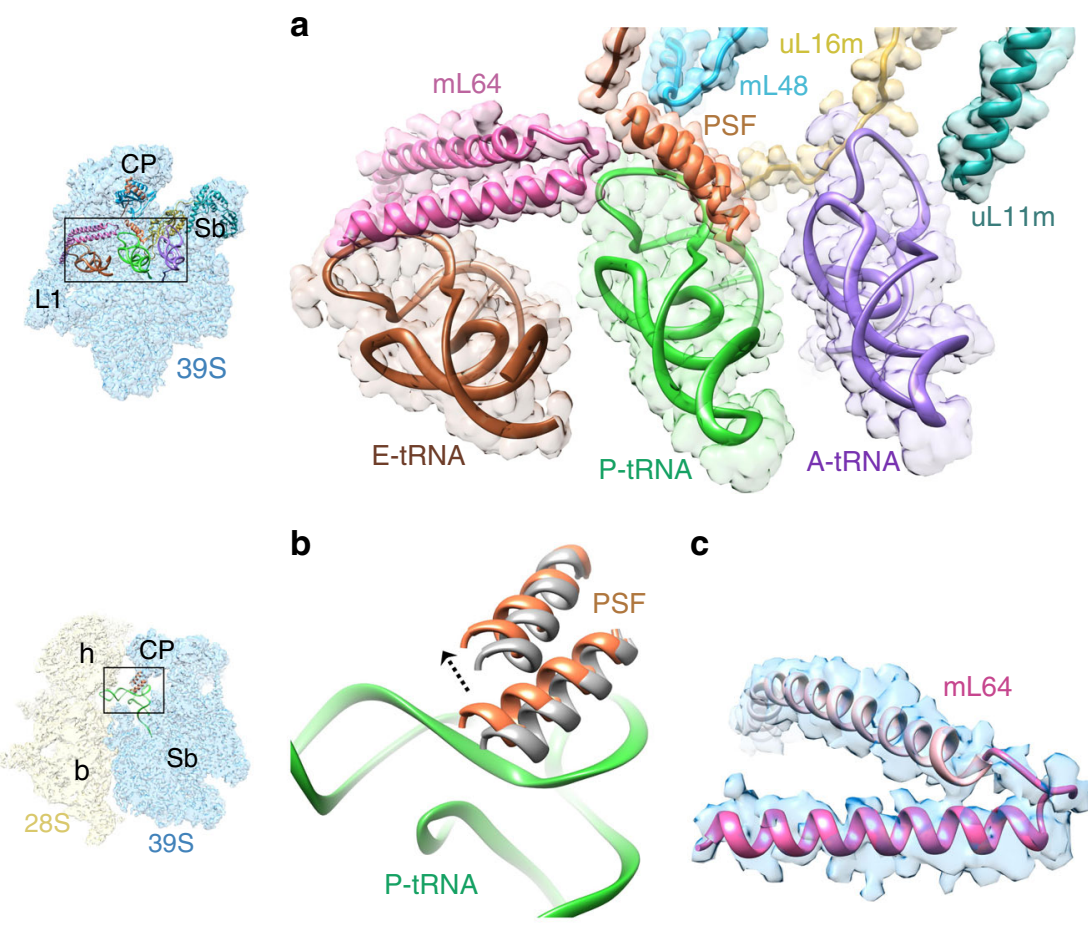

C

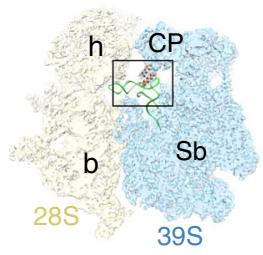

d
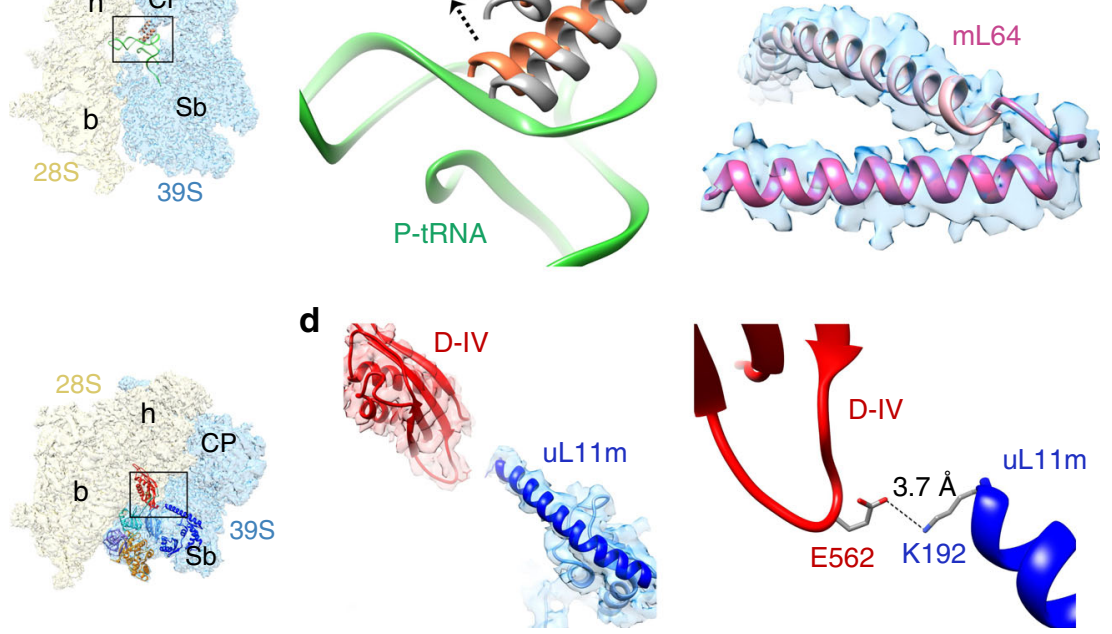

Fig. 4 Interactions of tRNAs $s_{\mathbf{m t}}$ and EF-G1 $\mathbf{m}_{\mathbf{m t}}$ with mito-specific proteins and mito-specific segments in homologous proteins. a Interactions of the $A$ (purple), P (green), and E (brown) tRNAs $s_{m t}$ with mito-specific proteins, PSF (orange), $\mathrm{mL} 48$ (blue), $\mathrm{mL64}$ (pink), and mito-specific insertion/extension to homologous proteins uL11m (dark cyan) and uL $16 \mathrm{~m}$ (yellow). The A-site tRNA $\mathrm{mt}_{\mathrm{t}}$ position in its A/A state is derived from the $55 \mathrm{~S}$ bovine (Bos taurus) map (see Supplementary Fig. 8). b Dynamics of PSF, which has moved by $\sim 4 \AA$ towards the P-site tRNA while interacting with the D and T loop regions P-site tRNA (P/P state) in the 55S.EF-G $\mathrm{mt}_{\mathrm{t}}$ GMPPCP complex, from its position in the vacant human mitoribosome (gray) ${ }^{2}$. c Structure of the C-terminal region (A160-E192, darker pink) of $\mathrm{mL} 64$ derived from Class III map (blue). $\mathbf{d}$ Interaction between the mito-specific segment of uL11m (dark blue) with the domain IV of EF-G1 mt (red), involving a hydrogen-bond between uL11m's C-terminal residue K192 and E562 from domain IV. Thumbnails to the left of panels $\mathbf{a}, \mathbf{b}$, and $\mathbf{d}$ depict overall orientation of the $39 \mathrm{~S}$ and $55 \mathrm{~S}$ mitoribosomes. Landmarks on thumbnails: CP central protuberance, L1 uL $1 \mathrm{~m}$ stalk, Sb uL $11 \mathrm{~m}$ stalk base of the $39 \mathrm{~S} \mathrm{LSU}$, h head, b body of the $28 \mathrm{~S}$ SSU.

after its translocation from the A to the $\mathrm{P}$ site. In comparison to empty 55 S mitoribosomes ${ }^{2,4}$, the PSF has undergone a significant conformational change and moved closer towards the P-site bound tRNA in all our EF-G1 $1_{m t}$-bound complexes (Fig. $4 \mathrm{~b}$ ) as well as in the mammalian mitochondrial initiation complex ${ }^{9}$. Tight interactions with the PSF could be one of the reasons for the frequent co-purification of mitoribosomes with a P-site bound $\mathrm{tRNA}_{\mathrm{mt}}{ }^{5}$.

In our maps, we found a previously unassigned tubular density in the region between the C-terminus of mito-specific protein mL64 and the PSF ${ }^{8}$. This extra density is readily attributable to an a-helix-forming 32 aa residues of the C-terminus of mL64 (Fig. 4c), which extends in the $39 \mathrm{~S}$ subunit between the P- and E-site tRNAs while interacting with the T-loop regions of both the tRNAs (Fig. 4a). Along with the mito-specific protein mL48 and the mito-specific segments of MRPs uL11m and uL16m, PSF and the C-terminus of mL64 span all three tRNA binding sites on the $39 \mathrm{~S}$ subunit (Fig. 4a), structurally compensating for the absence of some of the bacterial homologs of r-proteins and rRNA components that are known to be involved in tRNA positioning, stabilization and translocation in the bacterial ribosome.

In all three EF-G1 $1_{\mathrm{mt}}$-bound complexes the $\mathrm{uL} 11 \mathrm{~m}$ stalk-base region within the mitoribosomal LSU moves by $5 \AA$ towards the domain V of EF-G1 $1_{\mathrm{mt}}$ (Supplementary Fig. $4 \mathrm{a}, \mathrm{b}$ ), as compared to that in the empty $55 \mathrm{~S}$ mitoribosomes ${ }^{2,4}$, in the initiation ${ }^{9}$ and in mitoribosome recycling complexes ${ }^{8}$. A similar movement of the $\mathrm{uL} 11 \mathrm{~m}$ region was reported for the bacterial 70 S.EF-G complexes $^{29}$. However, in the mammalian mitoribosome the conformational change is associated with a direct contact between the domain IV of EF-G1 $1_{\mathrm{mt}}$ and the mito-specific segment of uL11m (Fig. 4d). K192 from the mito-specific C-terminus $\alpha$-helix of $\mathrm{uL} 11 \mathrm{~m}$ interacts with the E562 from the domain IV of EF-G1 $1_{\mathrm{mt}}$ through a hydrogen-bond interaction (Fig. 4d). Interestingly, the uniquely placed E562 is absent in EF-G2 $\mathrm{mt}$ (Supplementary Fig. 10). The presence of E562 and the mito-specific CTE in EF$\mathrm{G} 1_{\mathrm{mt}}$ and their absence in EF-G2 $2_{\mathrm{mt}}$, along with presence of four small insertion segments within corresponding domains II and III of EF-G2 $2_{\mathrm{mt}}$ (Supplementary Fig. 10), appear to be the key 

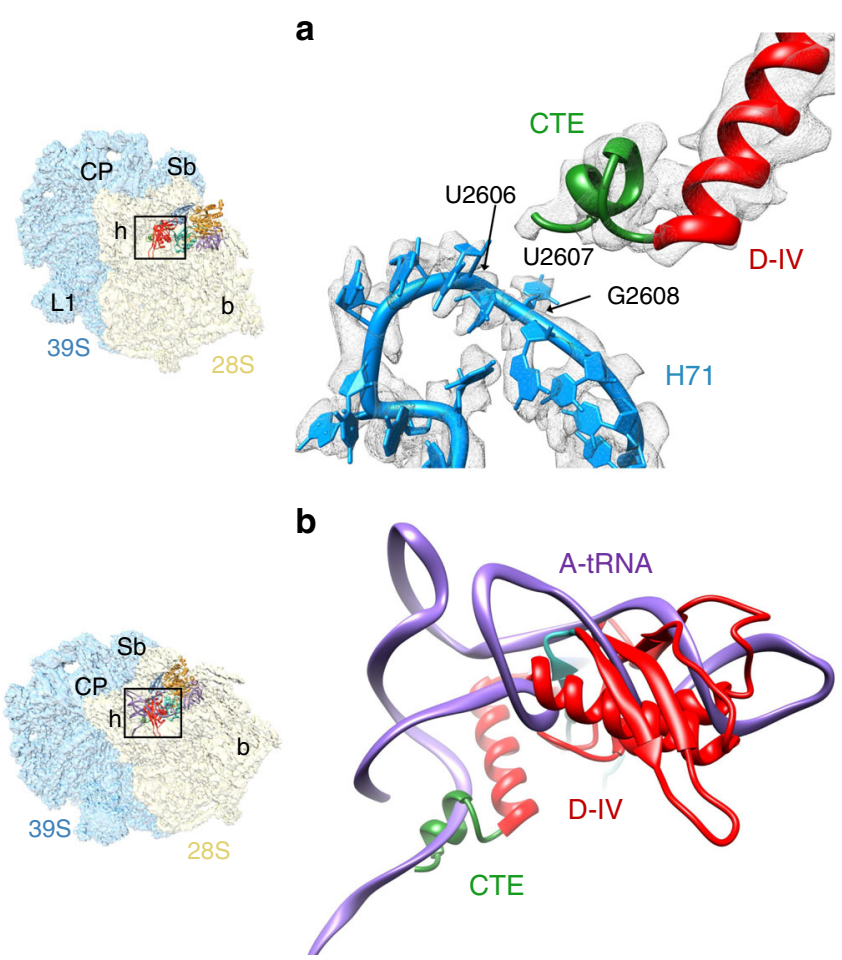

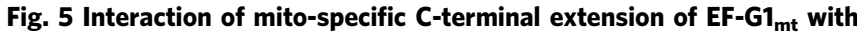
rRNA and tRNA. a The C-terminal extension (CTE, green) of domain IV (red) interacts with the 16S LSU rRNA helix 71 (H71, blue). b Superposition of the A-site bound tRNA $A_{m t}$ (purple) onto the EF-G1 $1_{m t}$-bound human $55 \mathrm{~S}$ structure revealed that while bulk of domain IV of EF-G1 ${ }_{\mathrm{mt}}$ overlaps with the anticodon-stem-loop region, the CTE partially overlaps with the CCA arm of the A-site tRNA $\mathrm{mt}_{\mathrm{m}}$. Landmarks on thumbnails to the left, which depict the overall orientations of the $55 \mathrm{~S}$ mitoribosome, are as in Fig. 4.

differences that confer specificity to these two factors for their roles in elongation and recycling steps, respectively.

Role of the C-terminal extension in EF-G1 $\mathbf{m}_{\mathbf{m t}}$. Both the Class I and Class III complexes show an additional density adjacent to the conserved C-terminal end of the EF-G1 mt domain IV that could readily accommodate its mito-specific C-terminal extension (CTE) (Fig. 5a), which is not resolved in the Class II complex. The lysine-rich CTE folds into an $\alpha$-helix and extends into the $39 \mathrm{~S}$ subunit enabling the EF-G1 $1_{\mathrm{mt}}$ to interact with rRNA and tRNA $_{m t}$ segments that would be inaccessible to the bacterial EFGs. The CTE is positioned close to the nucleotides U2606-G2608 segment of the 16S LSU rRNA helix 71 (H71) (Fig. 5a). In its current orientation, the CTE would overlap with the inner bend of A-site tRNA ${ }_{\mathrm{mt}}$ elbow primarily involving tRNA $\mathrm{mt}_{\mathrm{m}}$ 's CCA arm (Fig. 5b), suggesting that the CTE plays a direct role in the movement of the CCA arm of the A-site bound tRNA $\mathrm{mt}_{\text {. The }}$ interaction of lysine-rich CTE with $\mathrm{H} 71$ would also prevent the reverse translocation of the P-site tRNA to the A site. The fact that $\mathrm{EF}-\mathrm{G} 1_{\mathrm{mt}}$ remains active on the $E$. coli ribosomes, but $E$. coli EF-G remains inactive on mitoribosomes ${ }^{57}$, suggests that the observed interaction of EF-G1 ${ }_{\mathrm{mt}}$ 's CTE with the mitoribosome and the A-site tRNA $\mathrm{m}_{\mathrm{mt}}$ in our structure could also be associated with EF-G1 $1_{\mathrm{mt}}$ 's GTPase activity on the ribosome. A significantly altered landscape of the mitoribosomal intersubunit space described in the previous section and the location of EF-G1 $1_{\mathrm{mt}}$ 's CTE on the mitoribosome suggest that the MRPs and translational factors have coevolved with its unique tRNAs $\mathrm{mt}_{\mathrm{m}}$ to structurally and functionally compensate for the lost bacterial RNA segments.

Conformational changes at the nascent polypeptide-exit site. The newly synthesized protein chain exits the ribosome through a tunnel-like feature in the large subunit ${ }^{5,58,59}$ known as the nascent polypeptide-exit tunnel (NPET). The NPET originates from the peptidyltransferase center (PTC) and ends on the opposite side at the solvent interface, which is referred to as the polypeptide-exit site (PES). The structural composition of this tunnel is substantially different between the bacterial and the mammalian mitochondrial ribosomes ${ }^{2,4,5}$. Domains I and III of the $23 \mathrm{~S}$ rRNA that line the bottom portions of NPET in bacteria are greatly reduced in the analogous mitochondrial 16S LSU rRNA $^{5,53}$. The loss of these important structural components surrounding the tunnel is compensated through the acquisition of larger bacterial r-protein homologs with extended $\mathrm{N}$ and $\mathrm{C}$ termini ${ }^{5,54,56}$. A mito-specific protein $\mathrm{mL} 45$ is also present near the $\mathrm{PES}^{2,4}$. During the initiation phase, the entire NPET is blocked by the insertion of the N-terminus (NT) residues 38-64 of mL45 into the $\mathrm{NPET}^{9}$. The N-terminal region of mL45 also interacts with MRPs uL23m and uL24m near PES. Mutational studies have shown that deletion of the mL45 NT severely inhibits mitochondrial translation ${ }^{9}$.

Though the blocked NPET might not pose any problem for an initiating mitoribosome, a vacant tunnel would be necessary to accommodate the growing nascent polypeptide chain during the translation elongation phase. In all our complexes, we found an unassigned density that is connected to the CCA end of the P-site tRNA and reaches close to the NT of the ribosomal protein $\mathrm{mL} 45$ inside the NPET (Fig. 6a). This density that could accommodate up to 5 aa residues can be readily attributed to a nascent peptide chain (NPC). In our structure, a conserved adenine residue (A2725) from a loop region between the 16S LSU rRNA helices $\mathrm{H} 73$ and H74 intercalates between the NT of NPC and aa R40 from the NT of mL45 (Fig. 6a). By simultaneously interacting with the NPC and NT of mL45, A2725 might play a crucial role in triggering a conformational change in the large mitoribosomal subunit that eventually results in the retraction of NT of mL45 from the NPET to make room for the growing NPC. Indeed, we observed a significant conformational change involving aa residues R61 to D73 (Fig. 6b). Compared to their position in the initiation complex ${ }^{9}$, these residues have moved substantially, $\sim 9 \AA$ away from the tunnel and toward $\mathrm{uL} 24 \mathrm{~m}$, which also shifts in conjunction with the mL45 movement. The EF-G1 $1_{\mathrm{mt}}$-induced conformational change in the large subunit captured in our structure likely represents a functional state, as mL45 prepares to retrieve its NT from the NPET to allow the insertion of incoming nascent polypeptide from the PTC side of the NPET.

In addition, the residues T101-Y128 located in the core region of mL45 undergo a large conformational change (Fig. 6c) between the initiation ${ }^{9}$ and our elongation complexes. In the initiation complex, these residues form two separate $\alpha$-helices with an angle of $\sim 120^{\circ}$ between them. Of these, residues T109-T115 from the $\mathrm{N}$-terminal helix rotate by $\sim 60^{\circ}$ to become part of a single long a-helix in the elongation complex, leaving residues S101-R108 in an open conformation. This large conformational change, involving a secondary structure rearrangement, may be necessary for anchoring of the mammalian mitoribosome to the inner mitochondrial membrane (IMM). mL45 happens to be the homolog of Mba1, the IMM-associated receptor necessary for the co-translational insertion of nascent polypeptides into the IMM in yeast ${ }^{60}$. Interestingly, this positively charged segment (aa residues 101-114) of mL45 has been implicated in mediating the association of $55 \mathrm{~S}$ mitoribosomes with the negatively charged 
a
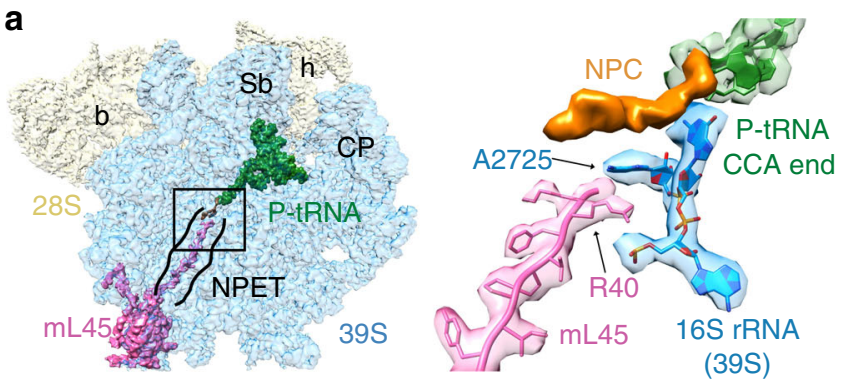

b
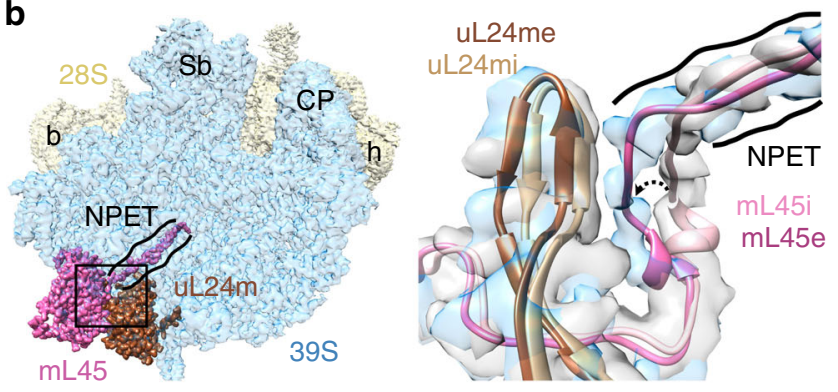

C
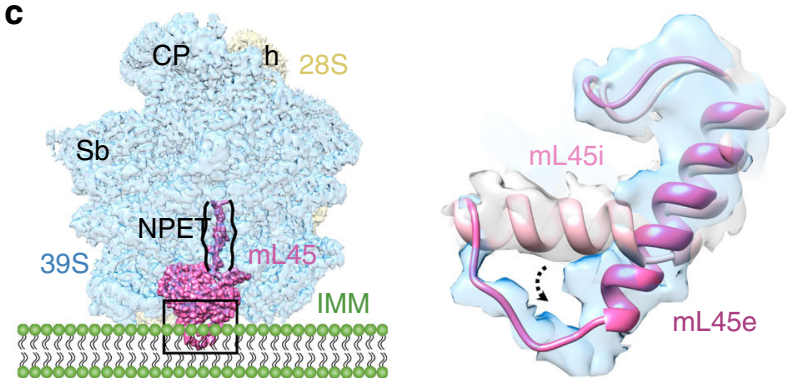

Fig. 6 Location of the nascent polypeptide chain and conformational changes in $\mathbf{m L} 45$ between the initiation and elongation complexes. a $A$ density linked to the CCA end of the P-site tRNA (green) was identified as NPC (orange) that approaches the N-terminus (NT) of ribosomal protein $\mathrm{mL} 45$ (pink). A2725 from the 16S LSU rRNA (blue) is positioned between the NT of $\mathrm{mL} 45$ and the nascent polypeptide chain (NPC). b The N terminus region (R61-D73) of $\mathrm{mL} 45$ in the elongation complex ( $\mathrm{mL} 45 \mathrm{e}$, deep pink) has shifted substantially toward $\mathrm{uL} 24 \mathrm{~m}$, as compared to its position in the initiation complex $(\mathrm{mL} 45 \mathrm{i} \text {, light pink })^{9}$. The shift in $\mathrm{mL} 45$ is accompanied by a small movement in uL24m between the initiation (uL24i, light brown) and elongation (uL24e, dark brown) states. c Conformational change in the core region of $\mathrm{mL} 45$. Curved arrows in panels $\mathbf{b}$ and $\mathbf{c}$ point to observed movements from initiation to elongation complexes. Thumbnails in panel $\mathbf{a}, \mathbf{b}$, and $\mathbf{c}$ depict the overall orientation of the $55 \mathrm{~S}$ mitoribosome where the 28S SSU (semitransparent yellow) is placed behind the 39S LSU (semitransparent blue), with relevant MRPs highlighted. Landmarks on the thumbnails are as in Fig. 4. Depiction of inner mitochondrial membrane (IMM) in panel c provides context to the location of the observed conformational change.

lipid content of the IMM through charge-based interactions ${ }^{61}$, which could be accompanied by the observed conformational change in this study. Furthermore, the C-terminal region of the $\mathrm{uL} 23 \mathrm{~m}$ that interacts with the mL45 NT has undergone a large conformational change where its $\alpha$-helix involving aa residues A123-R137 has moved by $\sim 20 \AA$ toward the mL45 NTE. It appears that this $\alpha$-helix movement is necessary to clear the path for the displacement of mL45's NTE from the exit tunnel (Supplementary Fig. 11a). However, a comparison of the sequences of $\mathrm{uL} 23 \mathrm{~m}$ in human with other mammals (Supplementary Fig. 11b) suggests that this major conformational difference could be species-specific.
In summary, our study presents the most complete structure for the human $55 \mathrm{~S}$ mitoribosome, and shows that the EF- $1_{\mathrm{mt}^{-}}$ bound mitoribosome can adopt at least three different conformations irrespective of the GTP hydrolysis state. The major variation occurs in the relative orientation of its entire $28 \mathrm{~S}$ subunit, or only its head domain, suggesting an unusual adaptability of the $28 \mathrm{~S}$ subunit during translocation (Fig. 3). Direct structural evidence is presented that the mito-specific components in both the mitoribosome and EF-G1 $1_{\mathrm{mt}}$ are involved in $\mathrm{tRNA}_{\mathrm{mt}}$ translocation. Our study also shows how mito-specific ribosomal proteins, such as PSF and mL64 in the mitoribosome's tRNA $\mathrm{mt}_{\mathrm{m}}$ interaction sites (Fig. 4), and the addition of a mere 11 aa residues in the Cterminus of EF-G1 $1_{\mathrm{mt}}$ (Fig. 5), allow the mitochondrial translation system to adapt to a massive reduction in mitoribosomal RNA components as compared to their bacterial counterparts. For example, the absence of $23 \mathrm{~S}$ rRNA helix 38 , also known as the Asite finger that dynamically interacts with both $\mathrm{A}$ - and P-site tRNAs during the tRNA translocation in eubacteria, is structurally and functionally compensated by the PSF protein in the mammalian mitoribosome. Similarly, the missing eubacterial Esite tRNA interacting rRNA components ${ }^{53}$ are replaced by protein mL64. Finally, the large conformational changes between the initiation and elongation states involving mito-specific protein mL45 in the NPET's exit site (Fig. 6), seem to be associated with the mitoribosomal anchoring to the IMM.

\begin{abstract}
Methods
Isolation of mitochondria from HEK cells. Mitochondria were isolated from human embryonic kidney cells lacking $N$-acetyl-glucosaminyltransferase I $(\mathrm{HEK} 293 \mathrm{~S} \mathrm{GnTI})^{8}$ that were cultured in roller bottles using FreeStyle $\mathrm{TM}_{293}$ media (Gibco, Life Technologies) supplemented with 5\% fetal bovine serum (Gibco, Life Technologies). After centrifugation at $1000 \times g$ for $7 \mathrm{~min}$, the HEK293S GnTI cellpellet was transferred to a glass homogenizer and resuspended in buffer containing $50 \mathrm{mM}$ HEPES-KOH pH 7.5, $10 \mathrm{mM} \mathrm{KCl,} 1.5 \mathrm{mM}$ MgOAc, $70 \mathrm{mM}$ sucrose, 210 mM mannitol, $1 \mathrm{mM}$ EDTA, $1 \mathrm{mM}$ EGTA, $1 \mathrm{mM}$ DTT, and $1 \mathrm{mM}$ PMSF. After homogenization, the supernatant was separated from the cell debris by spinning at $950 \times g$ for $15 \mathrm{~min}$. The supernatant was then spun at $11,000 \times g$ for $15 \mathrm{~min}$, and the resulting pellet that contains crude mitochondria was resuspended in SEM buffer (250 mM sucrose, $20 \mathrm{mM}$ HEPES-KOH pH 7.5, 1 mM EDTA, and $1 \mathrm{mM}$ EGTA). DNase I ( 3 units $/ \mathrm{ml}$ ) was added to the crude mitochondria and incubated at $4{ }^{\circ} \mathrm{C}$ for $1 \mathrm{~h}$. A discontinuous gradient was prepared in a Beckman polyallomer tube by layering $2.5 \mathrm{ml}$ of $60 \%, 4 \mathrm{ml}$ of $32 \%, 1 \mathrm{ml}$ of $23 \%$, and $1 \mathrm{ml}$ of $15 \%$ sucrose solutions in buffer containing $10 \mathrm{mM}$ HEPES-KOH pH 7.5 and $1 \mathrm{mM}$ EDTA. DNase-treated sample was loaded on the discontinuous gradient and centrifuged for $1 \mathrm{~h}$ at $135,000 \times g$ using Ti70 rotor in a Beckman ultracentrifuge. The brownish-orange layer containing pure mitochondria was carefully separated and re-suspended in
\end{abstract} SEM buffer.

Isolation of mitoribosomes from mitochondria. Mitoribosomes were isolated ${ }^{8}$ by adding four volumes of lysis buffer ( $25 \mathrm{mM}$ HEPES-KOH pH 7.5, $100 \mathrm{mM} \mathrm{KCl}, 25$ $\mathrm{mM}$ MgOAc, $1.7 \%$ Triton X-100, 2 mM DTT and $1 \mathrm{mM}$ PMSF) to the mitochondrial-pellet and then incubating for $15 \mathrm{~min}$ at $4{ }^{\circ} \mathrm{C}$. The sample was centrifuged at $30,000 \times g$ for $20 \mathrm{~min}$ and the supernatant was loaded on top of $1 \mathrm{M}$ sucrose cushion in buffer (20 mM HEPES-KOH pH 7.5, $100 \mathrm{mM} \mathrm{KCl,} 20 \mathrm{mM}$ MgOAc, $1 \%$ Triton X-100, and 2 mM DTT). After centrifugation for $17 \mathrm{~h}$ at $90,000 \times g$ using Ti70 rotor in Beckman ultracentrifuge, a minimal volume of Mitobuffer (20 mM HEPES-KOH pH 7.5, $100 \mathrm{mM} \mathrm{KCl,} 20 \mathrm{mM} \mathrm{MgOAc}$, and 2 mM DTT) enough to dissolve the pellet was added. $10-30 \%$ continuous sucrose density gradients were prepared in Mitobuffer, using the gradient making apparatus (C.B.S. Scientific Co.). The resuspended pellet was subjected to $10-30 \%$ continuous sucrose density gradient centrifugation at $60,000 \times g$ for $17 \mathrm{~h}$ using Sw32 rotor in Beckman ultracentrifuge. The gradient was fractionated on ISCO gradient analyzer (Teledyne ISCO, Inc), and the fractions corresponding to the mitoribosomes were collected and pooled. Finally, the pooled mitoribosomes were concentrated by spinning them at $130,000 \times g$ for $6 \mathrm{~h}$ using Ti70 rotor, and the pellet was resuspended in Polymix buffer $(5 \mathrm{mM}$ HEPES-KOH pH 7.5, $100 \mathrm{mM}$ $\mathrm{KCl}, 20 \mathrm{mM}$ MgOAc, $5 \mathrm{mM} \mathrm{NH}_{4} \mathrm{Cl}, 0.5 \mathrm{mM} \mathrm{CaCl}_{2}, 1 \mathrm{mM}$ DTT, $1 \mathrm{mM}$ spermidine, and $8 \mathrm{mM}$ putrescine $)^{62}$

Cloning and expression of human EF-G1 $\mathbf{m}_{\mathbf{m t}}$. An expressed sequence tag coding for human EF-G1 $1_{\mathrm{mt}}$ was obtained from GeneCopoeia (No. GC-W1058). Using PCR, the sequence corresponding to the mature form of EF-G1 $1_{\mathrm{mt}}$ (amino acids 36-751) was amplified by employing forward $5^{\prime}$-GGAATTCCATATGTCTTCATCAGGG GTGATTCC- $3^{\prime}$ and reverse $5^{\prime}$-AACCGCTCGAGTTCTTGGCTTTTCCTTTTTT 
AAC- $3^{\prime}$ primers ${ }^{39}$. The PCR product was cloned into pET 21c (+) (Novagen) and this vector provides a sequence encoding six His residues (His-tag) at the Cterminus. The resulting construct was transformed into E. coli ER2267 and subsequently transformed into E. coli BL21(DE3) (RIL) for over-expression.

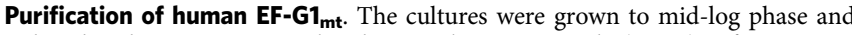
induced with $50 \mu \mathrm{M}$ isopropyl-1-thio-D-galactopyranoside (IPTG). After centrifugation at $5,000 \mathrm{rpm}$ for $15 \mathrm{~min}$ at $4{ }^{\circ} \mathrm{C}$, the cells were harvested, shock-frozen, and stored at $-80^{\circ} \mathrm{C}$. The frozen cells were disrupted by grinding with double the cell weight of Alumina Type A-5 (Sigma) for a total of $20 \mathrm{~min}$. The paste was resuspended in Buffer B (50 mM Tris- $\mathrm{HCl}$, pH 7.6, $40 \mathrm{mM} \mathrm{KCl}, 7 \mathrm{mM} \mathrm{MgCl}, 7$ $\mathrm{mM} \beta$-mercaptoethanol, $0.1 \mathrm{mM}$ phenylmethylsulfonyl fluoride, and $10 \%$ glycerol), and the debris was removed by centrifugation at $10,000 \mathrm{rpm}$ at $4{ }^{\circ} \mathrm{C}$ for $10 \mathrm{~min}$. This is followed by DNase I $(5 \mu \mathrm{g} / \mathrm{mL})$ treatment and centrifugation at $15,000 \mathrm{rpm}$ at $4{ }^{\circ} \mathrm{C}$ for $20 \mathrm{~min}$. The resulting supernatant was mixed with $0.6 \mathrm{~mL}$ of a $50 \%$ slurry of Ni-NTA resin equilibrated in Buffer B and relatively pure EF-G1 $1_{\mathrm{mt}}$ was obtained using affinity chromatography ${ }^{39}$. In order to achieve high-level purity, ion exchange chromatography technique was employed where the partially purified EF-G1mt from the Ni-NTA purification was processed on a cation exchange TSKgel SP-5PW column (TosoHaas, Japan).

\section{Preparation of the human mitoribosome $\bullet E F-G 1_{m t} \bullet$ GMPPCP complex. To} obtain the 55S.EF-G1 $1_{\mathrm{mt}}$ complex, a non-hydrolysable analog of GTP, GMPPCP, was used to lock EF-G1 $1_{\mathrm{mt}}$ on the mitoribosomes. The complex was formed by incubating $150 \mathrm{nM} 55 \mathrm{~S}$ mitoribosomes with $5 \mu \mathrm{M} \mathrm{EF}-\mathrm{Gl}_{\mathrm{mt}}$ and $1 \mathrm{mM}$ GMPPCP (Sigma-Aldrich, USA) at $37^{\circ} \mathrm{C}$ for $5 \mathrm{~min}$ in the HEPES polymix buffer.

Cryo-electron microscopy and image processing. In all, $4 \mu$ of the 55S.EF$\mathrm{G} 1_{\mathrm{mt}} \cdot \mathrm{GMPPCP}$ complex was applied to Quantifoil holey copper 1.2/1.3 grids that were pre-coated with a thin layer ( $\sim 50 \AA$ thick) of home-made continuous carbon film and glow-discharged for $30 \mathrm{~s}$ on a plasma sterilizer. After incubating the grids for $15 \mathrm{~s}$ at $4{ }^{\circ} \mathrm{C}$ and $100 \%$ humidity, they were blotted for $4 \mathrm{~s}$ and immediately flashfrozen into the liquid ethane with the help of a Vitrobot (FEI company). Data were collected on a Titan Krios electron microscope (FEI company) equipped with a Gatan K2 summit direct-electron detecting camera at $300 \mathrm{kV}$. We used a defocus range of -1.0 to $-3.0 \mu \mathrm{m}$ at a calibrated magnification of $\times 105,000$, yielding a pixel size of $1.0961 \AA$. A dose rate of 7 electrons per pixel per s and an exposure time of $10 \mathrm{~s}$ resulted in a total dose of $69.2 \mathrm{e} / \AA^{2}$. All the downstream processing of data was done using CryoSPARC ${ }^{63}$. Full-frame motion correction was applied to all 50 movie frames corresponding to each of the 6,671 micrographs. After determining their contrast transfer function (CTF) using CTFFIND4 $4^{64}$, bad images were deselected. From the remaining 6649 micrographs, 1,611,0847 particles were picked using the auto-pick function, and after local motion correction, 1,262,274 particles remained. This step was followed by the reference-free $2 \mathrm{D}$ classification and finally 851,131 good particles were retained based on the $2 \mathrm{D}$ averages. The initial 3D reconstruction and refinement yielded $2.74 \AA$ resolution $55 \mathrm{~S}$ map (Supplementary Fig. 1), with its 39 S subunit showing an overall resolution of $2.68 \AA$ (Supplementary Fig. 2), and the local resolution in the core regions of the 39S subunit were found to be in the 2.5-2.6 range (Supplementary Fig. 2). However, the $28 \mathrm{~S}$ subunit was relatively poorly resolved. Reference-based 3D classification was employed to separate $55 \mathrm{~S}$ mitoribosomes (289,982 particles) from $39 \mathrm{~S}$ subunits (408,686 particles) and $28 \mathrm{~S}$ subunits (152,463 particles). Particles corresponding to the $55 \mathrm{~S}$ mitoribosomes were further subjected to multiple rounds $3 \mathrm{D}$ classification that yielded three stable classes, Class I (99,804 particles), Class II (25,755 particles), and Class III (150,347 particles), and allowed us to remove a small population of 14,076 bad particles. After 3D refinement Class I, Class II and Class III yielded a final resolution of $2.97 \AA, 3.96 \AA$, and $2.96 \AA$, respectively, and all of the three classes showed strong densities that could be readily attributed to a bound EF$\mathrm{G1}_{\mathrm{mt}}$. After masked local refinements, the $28 \mathrm{~S}$ and $39 \mathrm{~S}$ subunits from the Class I complex were refined to $3.15 \AA$ and $2.91 \AA$, respectively; the $28 \mathrm{~S}$ and $39 \mathrm{~S}$ subunits from the Class II complex were refined to $4.82 \AA$ and $3.77 \AA$, respectively; and the $28 \mathrm{~S}$ and $39 \mathrm{~S}$ subunits from the Class III complex were refined to $3.04 \AA$ and $2.87 \AA$, respectively (Supplementary Fig. 2). The Gold-standard criterion of 0.143 FSC cutoff $^{65}$ was used to report all resolutions.

Model building and optimization. Coordinates corresponding to the small and large subunits of the published human mitoribosome (PDB ID: 3J9M) ${ }^{2}$ were used as the initial template. The higher resolution of our maps (Supplementary Fig. 3) enabled us to build multiple protein and rRNA segments that were not present in the previous human mitoribosome structures ${ }^{2,8,66}$. Highly resolved secondary structural elements (SSE) and amino acid side-chain features guided the manual building of the majority of protein models using UCSF Chimera $1.14^{67}$ and $\mathrm{COOT}^{68}$. For modeling the relatively low-resolved regions such as the L7/L12 stalk proteins and the $\mathrm{C}$ terminal domain (CTD) of L12 from the large subunit and protein $\mathrm{mS} 39$ from the small subunit, homologous structures from the porcine mitoribosome ${ }^{9}$ were used as a template. Additional segments ( 75 rRNA nts and 1082 aa residues) that were absent in the previous human mitoribosome structures were modeled de novo. These new rRNA segments were built in ModeRNA ser$v^{6}{ }^{69}$, using corresponding segments wherever available from the porcine mitoribosome $\mathrm{e}^{9}$ as a template. For building the P- and E-site tRNAs, the highresolution structure of yeast $\mathrm{RNNA}^{\text {Phe }}$ (PDB ID: $\left.1 \mathrm{EhZ}\right)^{70}$ was used as template to generate $t \mathrm{RNA}_{\mathrm{mt}}$ Phe. The $\mathrm{tRNA}_{\mathrm{mt}}$ Phe was docked manually and rigid body fitted into the corresponding cryo-EM density using Chimera $1.14^{67}$. Owing to suboptimal occupancies and inherent heterogeneity within the endogenously bound tRNAs $s_{m}$, the resolution corresponding to the $\mathrm{RNAA}_{\mathrm{mt}}$ densities were relatively low to allow any sequence-specific modeling of the P- and E-site tRNAs $s_{\mathrm{mt}}$. Homology models of EF-G1 $1_{\mathrm{mt}}$ generated in I-TASSER ${ }^{71}$ were used as the initial template. Regions in the homology model that do not fully accommodate into the corresponding EF-G1 $1_{\mathrm{mt}}$ density were modeled de novo using Chimera $1.14^{67}$ and $\mathrm{COOT}^{68}$. Lower resolution in our cryo-EM maps corresponding to the density of $\mathrm{EF}-\mathrm{G} 1_{\mathrm{mt}} \mathrm{C}$ terminal extension (CTE) restricted modeling of this region at the sidechain level but permitted building the carbon backbone guided by recognizable SSEs. For the final optimization of the models into the cryo-EM densities, we used the "Real-space refinement" function in PHENIX" 72 . The models were validated using Molprobity server ${ }^{73}$, and the overall statistics of EM reconstruction and molecular modeling are listed in Supplementary Table 1.

Reporting summary. Further information on research design is available in the Nature Research Reporting Summary linked to this article.

\section{Data availability}

The data that support this study are available from the corresponding authors upon reasonable request. The cryo-EM maps and atomic coordinates have been deposited in the Electron Microscopy and PDB Data Bank (wwPDB.org) under accession codes EMD21233 [https://www.ebi.ac.uk/pdbe/entry/emdb/EMD-21233] and PDB 6VLZ [https:// doi.org/10.2210/pdb6VLZ/pdb] for the EF-G1 mtt $^{-}$bound $55 \mathrm{~S}$ mitoribosome (Complex I), and EMD-21242 [https://www.ebi.ac.uk/pdbe/entry/emdb/EMD-21242] and PDB 6VMI

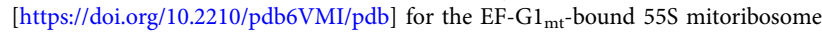
(Complex III). Cryo-EM maps of the Complex II and bovine 55S mitoribosome have been deposited with accession codes EMD-22212 [https://www.ebi.ac.uk/pdbe/entry/ emdb/EMD-22212] and EMD-22209 [https://www.ebi.ac.uk/pdbe/entry/emdb/EMD22209], respectively. All raw micrographs and particle images used in 3D reconstructions will be made available through empiar, an electron microscopy public image archive, https://www.ebi.ac.uk/pdbe/emdb/empiar/.

Received: 13 April 2020; Accepted: 15 July 2020;

Published online: 31 July 2020

\section{References}

1. Gray, M. W., Burger, G. \& Lang, B. F. The origin and early evolution of mitochondria. Genome Biol. 2, reviews1018.1-reviews1018.5 (2001).

2. Amunts, A., Brown, A., Toots, J., Scheres, S. H. W. \& Ramakrishnan, V. Ribosome. The structure of the human mitochondrial ribosome. Science 348, 95-98 (2015).

3. Desai, N., Brown, A., Amunts, A. \& Ramakrishnan, V. The structure of the yeast mitochondrial ribosome. Science 355, 528-531 (2017).

4. Greber, B. J. et al. Ribosome. The complete structure of the $55 \mathrm{~S}$ mammalian mitochondrial ribosome. Science 348, 303-308 (2015).

5. Sharma, M. R. et al. Structure of the mammalian mitochondrial ribosome reveals an expanded functional role for its component proteins. Cell 115, 97-108 (2003)

6. Florent Waltz, H. S., Bochler, A., Giegé, P., Hashem, Y. Cryo-EM structure of the RNA-rich plant mitochondrial ribosome. bioRxiv, https://doi.org/10.1101/ 777342 (2019).

7. Ramrath, D. J. F. et al. Evolutionary shift toward protein-based architecture in trypanosomal mitochondrial ribosomes. Science 362, eaau7735 (2018).

8. Koripella, R. K., Sharma, M. R., Risteff, P., Keshavan, P. \& Agrawal, R. K. Structural insights into unique features of the human mitochondrial ribosome recycling. Proc. Natl Acad. Sci. USA 116, 8283-8288 (2019).

9. Kummer, E. et al. Unique features of mammalian mitochondrial translation initiation revealed by cryo-EM. Nature 560, 263-267 (2018).

10. Christian, B. E. \& Spremulli, L. L. Mechanism of protein biosynthesis in mammalian mitochondria. Biochim. Biophys. Acta. 1819, 1035-1054 (2012).

11. Sharma, M. R., Kaushal, P. S., Gupta, M., Banavali, N. K. \& Agrawal, R. K. In Translation in Mitochondria and Other Organelles (ed. Duchêne A.-M.) Ch. 1, 1-28 (Springer, 2013)

12. Bhargava, K. \& Spremulli, L. L. Role of the N- and C-terminal extensions on the activity of mammalian mitochondrial translational initiation factor 3. Nucleic Acids Res. 33, 7011-7018 (2005).

13. Gaur, R. et al. A single mammalian mitochondrial translation initiation factor functionally replaces two bacterial factors. Mol. Cell 29, 180-190 (2008).

14. Haque, M. E. \& Spremulli, L. L. Roles of the N- and C-terminal domains of mammalian mitochondrial initiation factor 3 in protein biosynthesis. J. Mol. Biol. 384, 929-940 (2008). 
15. Rorbach, J. et al. The human mitochondrial ribosome recycling factor is essential for cell viability. Nucleic Acids Res. 36, 5787-5799 (2008).

16. Koripella, R. K. et al. Structure of human mitochondrial translation initiation factor 3 bound to the small ribosomal subunit. iScience 12, 76-86 (2019).

17. Yassin, A. S. et al. Insertion domain within mammalian mitochondrial translation initiation factor 2 serves the role of eubacterial initiation factor 1. Proc. Natl Acad. Sci. USA 108, 3918-3923 (2011).

18. Hammarsund, M. et al. Identification and characterization of two novel human mitochondrial elongation factor genes, hEFG2 and hEFG1, phylogenetically conserved through evolution. Hum. Genet. 109, 542-550 (2001).

19. Tsuboi, M. et al. EF-G2mt is an exclusive recycling factor in mammalian mitochondrial protein synthesis. Mol. Cell 35, 502-510 (2009).

20. Antonicka, H., Sasarman, F., Kennaway, N. G. \& Shoubridge, E. A. The molecular basis for tissue specificity of the oxidative phosphorylation deficiencies in patients with mutations in the mitochondrial translation factor EFG1. Hum. Mol. Genet. 15, 1835-1846 (2006).

21. Coenen, M. J. et al. Cytochrome $\mathrm{c}$ oxidase biogenesis in a patient with a mutation in COX10 gene. Ann. Neurol. 56, 560-564 (2004).

22. Abbott, J. A., Francklyn, C. S. \& Robey-Bond, S. M. Transfer, RNA. and human disease. Front. Genet. 5, 158 (2014).

23. Bugiardini, E. et al. MRPS 25 mutations impair mitochondrial translation and cause encephalomyopathy. Hum. Mol. Genet. 28, 2711-2719 (2019).

24. Pearce, S., Nezich, C. L. \& Spinazzola, A. Mitochondrial diseases: translation matters. Mol. Cell. Neurosci. 55, 1-12 (2013).

25. AEvarsson, A. et al. Three-dimensional structure of the ribosomal translocase: elongation factor $\mathrm{G}$ from Thermus thermophilus. EMBO J. 13, 3669-3677 (1994).

26. Czworkowski, J., Wang, J., Steitz, T. A. \& Moore, P. B. The crystal structure of elongation factor $\mathrm{G}$ complexed with GDP, at 2.7 A resolution. EMBO J. 13, 3661-3668 (1994).

27. Agrawal, R. K., Penczek, P., Grassucci, R. A. \& Frank, J. Visualization of elongation factor $\mathrm{G}$ on the Escherichia coli $70 \mathrm{~S}$ ribosome: the mechanism of translocation. Proc. Natl Acad. Sci. USA 95, 6134-6138 (1998).

28. Brilot, A. F., Korostelev, A. A., Ermolenko, D. N. \& Grigorieff, N. Structure of the ribosome with elongation factor $\mathrm{G}$ trapped in the pretranslocation state. Proc. Natl Acad. Sci. USA 110, 20994-20999 (2013).

29. Datta, P. P., Sharma, M. R., Qi, L., Frank, J. \& Agrawal, R. K. Interaction of the $\mathrm{G}$ ' domain of elongation factor $\mathrm{G}$ and the C-terminal domain of ribosomal protein L7/L12 during translocation as revealed by cryo-EM. Mol. Cell 20, 723-731 (2005).

30. Frank, J. \& Agrawal, R. K. A ratchet-like inter-subunit reorganization of the ribosome during translocation. Nature 406, 318-322 (2000).

31. Ramrath, D. J. et al. Visualization of two transfer RNAs trapped in transit during elongation factor G-mediated translocation. Proc. Natl Acad. Sci. USA 110, 20964-20969 (2013)

32. Ratje, A. H. et al. Head swivel on the ribosome facilitates translocation by means of intra-subunit tRNA hybrid sites. Nature 468, 713-716 (2010)

33. Agrawal, R. K., Heagle, A. B., Penczek, P., Grassucci, R. A. \& Frank, J. EF-Gdependent GTP hydrolysis induces translocation accompanied by large conformational changes in the 70S ribosome. Nat. Struct. Biol. 6, 643-647 (1999).

34. Chen, Y., Feng, S., Kumar, V., Ero, R. \& Gao, Y. G. Structure of EF-Gribosome complex in a pretranslocation state. Nat. Struct. Mol. Biol. 20, 1077-1084 (2013).

35. Gao, Y. G. et al. The structure of the ribosome with elongation factor G trapped in the posttranslocational state. Science 326, 694-699 (2009).

36. Tourigny, D. S., Fernandez, I. S., Kelley, A. C. \& Ramakrishnan, V. Elongation factor $\mathrm{G}$ bound to the ribosome in an intermediate state of translocation. Science 340, 1235490 (2013).

37. Zhou, J., Lancaster, L., Donohue, J. P. \& Noller, H. F. Crystal structures of EFG-ribosome complexes trapped in intermediate states of translocation. Science 340, 1236086 (2013).

38. Zhou, J., Lancaster, L., Donohue, J. P. \& Noller, H. F. How the ribosome hands the A-site tRNA to the P site during EF-G-catalyzed translocation. Science 345, 1188-1191 (2014).

39. Bhargava, K., Templeton, P. \& Spremulli, L. L. Expression and characterization of isoform 1 of human mitochondrial elongation factor G. Protein Expr. Purif. 37, 368-376 (2004).

40. Diaconu, M. et al. Structural basis for the function of the ribosomal L7/12 stalk in factor binding and GTPase activation. Cell 121, 991-1004 (2005).

41. Helgstrand, M. et al. The ribosomal stalk binds to translation factors IF2, EFTu, EF-G and RF3 via a conserved region of the L12 C-terminal domain. J. Mol. Biol. 365, 468-479 (2007).

42. Rodnina, M. V., Peske, F., Peng, B. Z., Belardinelli, R. \& Wintermeyer, W. Converting GTP hydrolysis into motion: versatile translational elongation factor G. Biol. Chem. 401, 131-142 (2019).
43. Rodnina, M. V., Savelsbergh, A., Katunin, V. I. \& Wintermeyer, W. Hydrolysis of GTP by elongation factor G drives tRNA movement on the ribosome. Nature 385, 37-41 (1997).

44. Cool, R. H. \& Parmeggiani, A. Substitution of histidine- 84 and the GTPase mechanism of elongation factor Tu. Biochemistry 30, 362-366 (1991).

45. Daviter, T., Wieden, H. J. \& Rodnina, M. V. Essential role of histidine 84 in elongation factor Tu for the chemical step of GTP hydrolysis on the ribosome. J. Mol. Biol. 332, 689-699 (2003)

46. Frank, J. \& Agrawal, R. K. Ratchet-like movements between the two ribosomal subunits: their implications in elongation factor recognition and tRNA translocation. Cold Spring Harb. Symp. Quant. Biol. 66, 67-75 (2001).

47. Schuwirth, B. S. et al. Structures of the bacterial ribosome at $3.5 \mathrm{~A}$ resolution Science 310, 827-834 (2005)

48. Kaushal, P. S., Sharma, M. R. \& Agrawal, R. K. The 55 S mammalian mitochondrial ribosome and its tRNA-exit region. Biochimie 114, 119-126 (2015).

49. Savelsbergh, A., Matassova, N. B., Rodnina, M. V. \& Wintermeyer, W. Role of domains 4 and 5 in elongation factor $\mathrm{G}$ functions on the ribosome. J. Mol. Biol. 300, 951-961 (2000).

50. Hanada, T. et al. Translation ability of mitochondrial tRNAsSer with unusual secondary structures in an in vitro translation system of bovine mitochondria. Genes Cells 6, 1019-1030 (2001).

51. Ohtsuki, T., Kawai, G. \& Watanabe, K. The minimal tRNA: unique structure of Ascaris suum mitochondrial tRNA(Ser)(UCU) having a short T arm and lacking the entire D arm. FEBS Lett. 514, 37-43 (2002).

52. Pulk, A. \& Cate, J. H. Control of ribosomal subunit rotation by elongation factor G. Science 340, 1235970 (2013).

53. Mears, J. A. et al. A structural model for the large subunit of the mammalian mitochondrial ribosome. J. Mol. Biol. 358, 193-212 (2006).

54. Koc, E. C. et al. The large subunit of the mammalian mitochondrial ribosome. Analysis of the complement of ribosomal proteins present. J. Biol. Chem. 276 43958-43969 (2001).

55. O'Brien, T. W. Evolution of a protein-rich mitochondrial ribosome: implications for human genetic disease. Gene 286, 73-79 (2002)

56. Suzuki, T. et al. Structural compensation for the deficit of rRNA with proteins in the mammalian mitochondrial ribosome. Systematic analysis of protein components of the large ribosomal subunit from mammalian mitochondria. J. Biol. Chem. 276, 21724-21736 (2001).

57. Eberly, S. L., Locklear, V. \& Spremulli, L. L. Bovine mitochondrial ribosomes. Elongation factor specificity. J. Biol. Chem. 260, 8721-8725 (1985).

58. Ban, N., Nissen, P., Hansen, J., Moore, P. B. \& Steitz, T. A. The complete atomic structure of the large ribosomal subunit at $2.4 \mathrm{~A}$ resolution. Science 289, 905-920 (2000).

59. Frank, J. et al. A model of protein synthesis based on cryo-electron microscopy of the E. coli ribosome. Nature 376, 441-444 (1995).

60. Lorenzi, I. et al. Ribosome-associated Mba1 escorts Cox2 from insertion machinery to maturing assembly intermediates. Mol. Cell Biol. 36, 2782-2793 (2016).

61. Englmeier, R., Pfeffer, S. \& Forster, F. Structure of the human mitochondrial ribosome studied in situ by cryoelectron tomography. Structure 25, 1574-1581 (2017).

62. Koripella, R. K. et al. A conserved histidine in switch-II of EF-G moderates release of inorganic phosphate. Sci. Rep. 5, 12970 (2015).

63. Punjani, A., Rubinstein, J. L., Fleet, D. J. \& Brubaker, M. A. CryoSPARC: algorithms for rapid unsupervised cryo-EM structure determination. Nat. Methods 14, 290-296 (2017).

64. Rohou, A. \& Grigorieff, N. CTFFIND4: fast and accurate defocus estimation from electron micrographs. J. Struct. Biol. 192, 216-221 (2015).

65. Rosenthal, P. B. \& Henderson, R. Optimal determination of particle orientation, absolute hand, and contrast loss in single-particle electron cryomicroscopy. J. Mol. Biol. 333, 721-745 (2003).

66. Brown, A. et al. Structure of the large ribosomal subunit from human mitochondria. Science 346, 718-722 (2014).

67. Pettersen, E. F. et al. UCSF Chimera-a visualization system for exploratory research and analysis. J. Comput. Chem. 25, 1605-1612 (2004).

68. Emsley, P., Lohkamp, B., Scott, W. G. \& Cowtan, K. Features and development of Coot. Acta Crystallogr. D Biol. Crystallogr. 66, 486-501 (2010).

69. Rother, M. et al. ModeRNA server: an online tool for modeling RNA 3D structures. Bioinformatics 27, 2441-2442 (2011).

70. Shi, H. \& Moore, P. B. The crystal structure of yeast phenylalanine tRNA at 1.93 A resolution: a classic structure revisited. RNA 6, 1091-1105 (2000).

71. Roy, A., Kucukural, A. \& Zhang, Y. I-TASSER: a unified platform for automated protein structure and function prediction. Nat. Protoc. 5, 725-738 (2010). 
72. Adams, P. D. et al. PHENIX: a comprehensive Python-based system for macromolecular structure solution. Acta Crystallogr. D Biol. Crystallogr. 66 213-221 (2010).

73. Chen, V. B. et al. MolProbity: all-atom structure validation for macromolecular crystallography. Acta Crystallogr. D Biol. Crystallogr. 66, 12-21 (2010)

\section{Acknowledgements}

We thank ArDean Leith for help with computation. We acknowledge the use of the Wadsworth Center's Media and Culture core facility, for help in producing large volumes of HEK293S GnTI cells, and the Wadsworth Center's and New York Structural Biology Center's (NYSBC's) EM facilities. NYSBC EM facilities are supported by grants from the Simons Foundation (349247), NYSTAR, the NIH (GM103310), and the Agouron Institute (F00316). This work was supported by the NIH grant R01 GM61576 (to R.K.A.).

\section{Author contributions}

R.K.A. conceived this study. K.B. and L.L.S. contributed to reagents, including purified EF-G1 $1_{\mathrm{mt}}$ and clones for EF-G1 $1_{\mathrm{mt}}$; P.K. purified human mitoribosomes and human EF-G1 $1_{\mathrm{mt}}$; R.K.K. prepared the human 55S-EF-G1 $1_{\mathrm{mt}}$ complex. R.K.K., M.R.S., and N.K.B. collected cryo-EM data and performed image processing. M.R.S., P.P.D., and P.S.K. performed purification and reconstructions of bovine mitoribosomes, R.K.K., M.R.S., and N.K.B. performed molecular modeling, R.K.K., M.R.S., and R.K.A. analyzed the data and wrote the manuscript.

\section{Competing interests}

The authors declare no competing interests.

\section{Additional information}

Supplementary information is available for this paper at https://doi.org/10.1038/s41467020-17715-2.

Correspondence and requests for materials should be addressed to R.K.A.

Peer review information Nature Communications thanks the anonymous reviewers for their contribution to the peer review of this work. Peer reviewer reports are available.

Reprints and permission information is available at http://www.nature.com/reprints

Publisher's note Springer Nature remains neutral with regard to jurisdictional claims in published maps and institutional affiliations.

(c) (i) Open Access This article is licensed under a Creative Commons Attribution 4.0 International License, which permits use, sharing, adaptation, distribution and reproduction in any medium or format, as long as you give appropriate credit to the original author(s) and the source, provide a link to the Creative Commons license, and indicate if changes were made. The images or other third party material in this article are included in the article's Creative Commons license, unless indicated otherwise in a credit line to the material. If material is not included in the article's Creative Commons license and your intended use is not permitted by statutory regulation or exceeds the permitted use, you will need to obtain permission directly from the copyright holder. To view a copy of this license, visit http://creativecommons.org/ licenses/by/4.0/.

(C) The Author(s) 2020 